\title{
Fishing Spider Cluster Configuration: An Energy- Efficient Wireless Sensor Clustering Method
}

\author{
Kyuhong Lee and Heesang Lee* \\ Department of Systems Management Engineering, Sungkyunkwan University, \\ Suwon 135-842, Korea
}

(Received November 5, 2012; accepted February 4, 2013)

Key words: wireless sensor network, energy efficiency, self-organized, bioinspired clustering model

In this paper, we propose an energy-efficient clustering model for wireless sensor networks. This model performs self-organized clustering using local information and simple rules inspired by the behaviors and capabilities of the six-spotted fishing spider Dolomedes triton. We compare our model with a well-known cluster-based routing protocol that uses random fairness for the selection of cluster heads. In our computational experiments, we show that the energy efficiency, lifetime, and scalability of our model exceed those of the comparison protocol.

\section{Introduction}

In the biological world, living organisms are observed in order to determine their behavior patterns. As many living organisms exhibit simple behavior patterns, bioinspired mechanisms often exhibit characteristics of self-organization.

Self-organization has numerous advantages over classical clustering and routing models in telecommunication networks. ${ }^{(1,2)}$ The first advantage is simplicity, i.e., the ability to overcome weak capacities of the sensor nodes in a network. The second advantage is scalability, and the third advantage is adaptability, which is the capability to adapt to changes in the environment.

Wireless sensor networks (WSNs) have various characteristics and inherent constraints. ${ }^{(3-8)}$ First, a WSN consists of hundreds or thousands of wireless sensor nodes. Second, data that are sensed from the entire set of wireless sensor nodes must ultimately reach the base station (BS). Third, to ensure their cost-effectiveness, each sensor node in a WSN is constrained in terms of energy, processing capability, and storage capacity. ${ }^{(9)}$ These characteristics must be taken into consideration when developing a clustering and routing model for a WSN.

As sensor nodes have weak capabilities and WSNs operate under various constraints, simple mechanisms are better accommodated than complex mechanisms. Therefore, a

${ }^{*}$ Corresponding author: e-mail: leehee@skku.edu 
mechanism based on bioinspired approaches may be appropriate, particularly for WSNs. In this article, we propose an energy-efficient clustering model using an approach inspired by the fishing spider Dolomedes triton.

\section{Bioinspired Rules Derived from the Fishing Spider Dolomedes triton}

Our primary idea was derived from the behavior of the six-spotted fishing spider $D$. triton. This spider lives near small lakes and ponds, and hunts its prey on the surface of the water. Fishing spiders extend their front legs in order to feel vibrations on the surface of the water. This is similar to other land-based spiders, who feel the vibrations made by an insect trapped in a web. As fishing spiders have a range of vibration-detecting organs, including very sensitive hairs (trichobothria) on their legs and feet, they can identify the source of the vibrations and also discern the distance from and direction of the source. ${ }^{(10-12)}$

In order to mate, male fishing spiders must communicate with neighboring female fishing spiders. ${ }^{(10)}$ A male will jerk his body and generate concentric surface waves, which have characteristic frequencies that differentiate him from prey, for the neighboring females. ${ }^{(12)}$ Females are able to classify water surface waves according to their frequency and duration. If a wave is long-lasting and has a frequency above $50 \mathrm{~Hz}$, the female recognizes the object as prey.

In our fishing spider-inspired WSN clustering, a sensor node locates the neighborhood sensor nodes by capturing the concentric radio signals broadcast by the neighboring sensor nodes. Sensor nodes count the number of signals received from neighboring nodes that broadcast their signals. Each neighboring sensor node can be differentiated from other neighbor sensor nodes by identifying the information; this is similar to the frequencies and durations of water surface waves for fishing spiders. A sensor node can discriminate between various types of radio signal for clustering and data transmission.

\section{Network Modeling}

\subsection{Definitions for the model}

In this article, we propose a fishing spider cluster configuration (FS-CC) protocol. We first define the following terms. In the data transmission phase, a cluster member (CM) creates a data packet, and transmits that packet to the BS via its cluster head $(\mathrm{CH})$. A cycle of that process is defined as a "period". The number of periods carried out within a data transmission phase is defined as a "round". We assume that each round is composed of 10 periods. We define the network lifetime of the WSN as the point at which $20 \%$ of the sensor nodes are drained.

\subsection{States of sensor nodes}

Each sensor node in the FS-CC performs its duties while in one of the following five states, which are dependent on the roles of the sensor node.

Undecided state: The node does not belong to any cluster.

CH candidate state: The node competes to become a $\mathrm{CH}$.

CH state: The node collects and aggregates information from its CMs and transfers the 
data packets to the BS.

CM state: The node periodically sends information to its $\mathrm{CH}$.

Drained state: The node cannot function as its energy has been drained; it no longer participates in the network.

\section{Fishing-Spider-Inspired Clustering}

FS-CC comprises a clustering phase that forms the clusters and a data transmission phase that sends/receives the data packets. FS-CC determines the CHs in every round, and operates by utilizing local information and several simple rules.

\subsection{Clustering phase}

This phase commences when the sensor nodes are first scattered in the sensor field, or after the data transmission phase has been completed.

Broadcasting Step: At period zero, all of the sensor nodes are assumed to be in the same cluster. In order to choose the next $\mathrm{CH}$, the $\mathrm{CHs}$ broadcast a $\mathrm{CH}$-change-signal packet within a predetermined broadcasting range. This is similar to the behavior of male fishing spiders when generating concentric water surface waves. By limiting the broadcasting range, the overhead of the clustering phase can be reduced; this is due to the proximity of nodes in the $\mathrm{CH}$ candidate state.

CH Selection Step: Every node in the $\mathrm{CH}$ candidate state broadcasts a $\mathrm{CH}$-candidate signal packet within the broadcasting range, and counts the signal packets received from the same cluster. This is similar to the process wherein a fishing spider recognizes and classifies each of the received surface signals from neighboring fishing spiders according to their frequency and duration. Every $\mathrm{CH}$ candidate node broadcasts a $\mathrm{CH}$-candidate information signal packet over twice the broadcasting range in order to obtain energy state information about $\mathrm{CH}$-candidate states in the same cluster.

Clustering Step: In order to organize its cluster, each $\mathrm{CH}$ broadcasts a $\mathrm{CH}$ signal packet within the broadcasting range. The sensor nodes that receive signal packets, except the $\mathrm{CH}$ or nodes in the drained state, select the nearest $\mathrm{CH}$ and become the $\mathrm{CMs}$ of that cluster.

\subsection{Data transmission phase}

As soon as the clusters are organized, information sensed by each sensor node is transmitted to the BS by sending data packets during the data transmission phase. Each $\mathrm{CH}$ aggregates the received data packets from its CMs and directly transmits the aggregated data packet to the BS. Data transmission is carried out using the same cluster configuration during each round.

\subsection{Fitness value}

The most important factor affecting the clustering performance is the $\mathrm{CH}$ selection method. In order to select the appropriate $\mathrm{CHs}$, FS-CC uses the following fitness comparison procedure.

First, FS-CC uses the number of neighbor nodes in the $\mathrm{CH}$-candidate state in order to 
check the appropriateness of the location of a $\mathrm{CH}$ :

$$
\text { Neighborhood degree fitness of } v=\left(1+\frac{\text { Number of neighbors of } v}{\text { Max. number of neighbors of cluster }(v)}\right)^{\alpha}
$$

where cluster $(v)$ means the cluster containing the sensor node $v$ and $\alpha$ is the average ratio of the remaining energy to the initial energy of cluster $v$.

Second, as $\mathrm{CH}$ consumes more energy than the $\mathrm{CMs}$, FS-CC gives priority to the $\mathrm{CH}$ candidate nodes with more residual energy:

$$
\text { Energy state fitness of } v=\left(1+\frac{\text { Residual energy of } v}{\text { Max. residual energy of cluster }(v)}\right)^{\alpha}
$$

The fitness value is the sum of the values of eqs. (1) and (2). We use $\alpha$ and $1-\alpha$ as exponent parameters; these reflect the state of the network as well as changes in the environment. Initially, the energy state is not important. However, as time passes, the energy state should be considered in order to lengthen the lifetime of the WSN.

\section{Experiments}

In this section, we show the performance of FS-CC. The performance of FS$\mathrm{CC}$ is compared with that of low energy adaptive clustering hierarchy (LEACH), ${ }^{(13)}$ a well-known self-organized clustering and routing method. In the LEACH protocol, sensor nodes are selected at random to be CHs. Random selection is good for the selforganization of the cluster configuration, but does not fully exploit energy efficiency.

\subsection{Basic comparisons}

We implemented FS-CC and LEACH using Visual C++ in Visual Studio 2008. One hundred sensor nodes with an initial energy of $0.5 \mathrm{~J}$ were distributed in a $50 \times 50 \mathrm{~m}^{2}$ sensor field. We fixed the location of the BS (25 and $150 \mathrm{~m})$, and assumed data and signal packet sizes of 1000 and 50 bits, respectively. In the test, the average performance of 10 different sensor distributions was used. We used a first-order radio model ${ }^{(13)}$ to simulate energy consumption.

First, we compared the lifetimes of FS-CC and LEACH. Figure 1 shows that the lifetimes of FS-CC were 22.9-47.5\% longer than those of LEACH. Second, we compared the residual energies of FS-CC and LEACH. Figure 2 shows the maximum, average, and minimum residual energies of the 100 sensor nodes for FS-CC and LEACH. We can see that the energy consumption of the sensor node with the minimum residual energy was better maintained by FS-CC. This is because FS-CC reflects the state of the network and considers the energy level of each sensor node. As time passes, FS-CC prefers to use sensor nodes with high energy levels, such as $\mathrm{CHs}$, and thus, the sensor nodes maintain longer network lifetimes. 


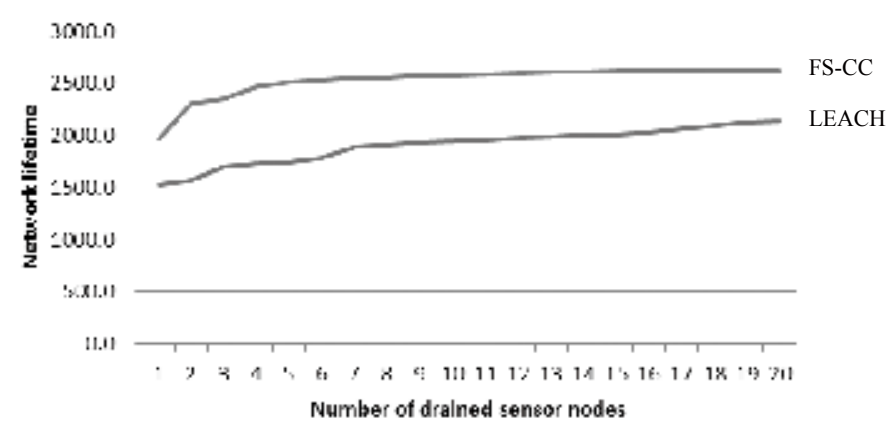

Fig. 1. Comparison of network lifetimes of FS-CC and LEACH.

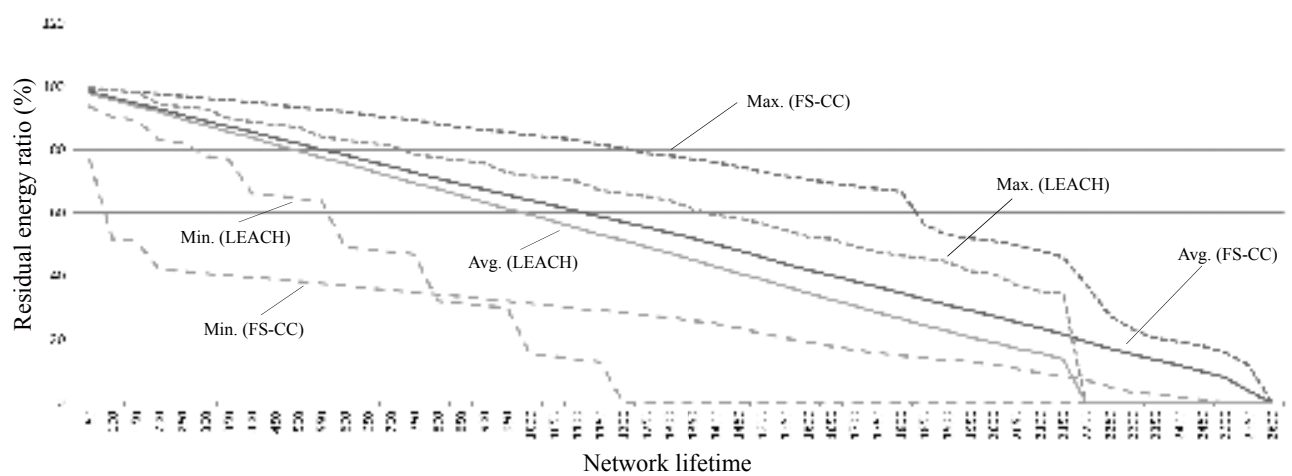

Fig. 2. Residual energy ratios of FS-CC and LEACH.

From the two experiments, we can conclude that FS-CC ensures a longer network lifetime than LEACH, and that the sensor nodes in FS-CC consume energy more uniformly than those in LEACH. This is because FS-CC controls certain sensor nodes; thus, it conserves energy by balancing the energy consumption of the sensor nodes based on the appropriate fitness value.

\subsection{Comparison of scalability}

We compared the network lifetimes of FS-CC and LEACH for sensor fields of different sizes and different numbers of sensor nodes; however, we maintained the same node density. Figure 3 shows that the network lifetimes of FS-CC were 22.9$58.5 \%$ longer than that of LEACH. Hence, FS-CC shows good scalability, enabling it to organize suitable clusters for different sensor field sizes. 


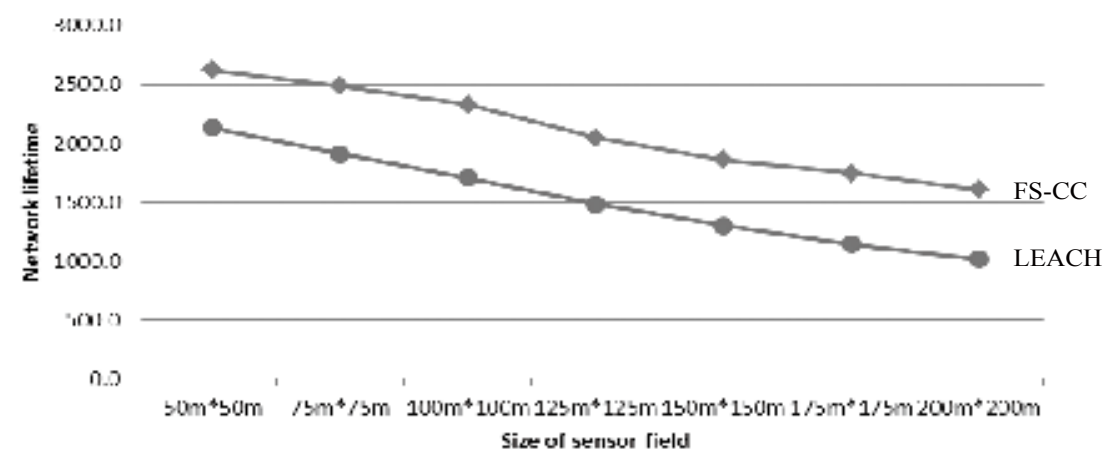

Fig. 3. Experiment on scalability of FS-CC and LEACH.

\section{Conclusions}

In this article, we have suggested a new bioinspired model for energy-efficient routing in WSNs. We used a clustering approach that organized sensor nodes into clusters and selected the CHs. In our experiments, the FS-CC was shown to use the energy of the sensor nodes efficiently, resulting in a prolonged WSN lifetime. We also proved that our FS-CC is scalable, which is important for self-organized WSN systems.

\section{Acknowledgements}

This work was supported by the Korea Research Foundation Grant funded by the Korean Government (KRF 313-2008-2-D01208)).

\section{References}

1 C. Prehofen and C. Bettstetter: IEEE Commun. Mag. 43 (2005) 78.

2 F. Dressler: Comput. Commun. 31 (2008) 3018.

3 J. N. Al-Karaki and A. E. Kamal: IEEE Wireless Commun. 11 (2004) 6.

4 K. Akkaya and M. Younis: ELSVIER Ad Hoc Networks 3 (2003) 325.

5 Y. Zhang, G. Sun and W. Li: Sens. Lett. 9 (2011) 1855.

6 L. T. Grigorie and R. M. Botez: Trans. Can. Soc. Mech. Eng. 34 (2010) 1.

7 L. Zhou, Y. Yang and F. G. Yuan: J. Vibroeng. 14 (2012) 280.

8 A. M. A. Soliman, M. M. S. Kaldas, D. C. Barton and P. C. Brooks: Int. Eng. Technol. Innovation 2 (2012) 85.

9 T. Nakamoto, T. Ikeda, H. Hirano and T. Arimoto: Sens. Mater. 23 (2011) 71.

10 H. Bleckmann and M. Bender: J. Arachnol. 15 (1987) 363.

11 H. Bleckmann and F. G. Barth: Behav. Ecol. Sociobiol. 14 (1984) 303.

12 C. Roland and J. S. Rovner: J. Arachnol. 11 (1983) 77.

13 W. R. Heinzelman, A. Chandrakasan and H. Balakrishnan: Proc. 33rd Annu. Hawaii Int. Conf. IEEE, 2000, p. 1. 